\title{
On the WM Points of Orlicz Function Spaces Endowed with Luxemburg Norm*
}

\author{
Tingfu WANG, Cuixia HAO and Minli LI
}

\begin{abstract}
The concept of WM point is introduced and the criterion of WM property in Orlicz function spaces endowed with Luxemburg norm is given,
\end{abstract}

It is well known that WM property is an important property in Geometry of Orlicz Spaces. The criteria of WM property have been discussed. [1-3] In this paper, we introduce the concept of WM point and give a criterion of WM point in Orlicz function spaces $L_{M}$ endowed with Luxemburg norm. Hence, we get easily a sufficient and necessary condition that $L_{M}$ has WM property.

Let $X$ be a Banach Space, $B(X), S(X)$ be the unit ball and unit sphere of $X$, respectively. $x \in S(X)$ is called WM point provided $x_{n}{ }^{\prime} \epsilon$ $B(X)$ and $\left\|x_{n}+x\right\| \rightarrow 2$ imply that there exists a supporting functional

1991 Mathematics Subject Classification: 46B30

Servicio publicaciones Univ. Complutense. Madrid, 1995.

${ }^{*}$ The subject supported by NSFC and NSFH. 
$f$ at $x$ such that $f\left(x_{n}\right) \rightarrow 1$. If every $x \in S(X)$ is WM point, then we say that $X$ has WM property.

$M(u), N(v)$ denote a pair of complemented $N$-functions, $p_{-}(u)$ and $p(u)$ denote the left and right derivatives of $M(u)$, respectively. $S_{M}$ denote a set of strictly convex points. $[a, b]$ is called a structural affine interval of $M(u)$, if $M(u)$ is linear on $[a, b]$, and for any $\varepsilon>0, M(u)$ is neither linear on $[a-\varepsilon, b]$ nor on $[a, b+\varepsilon]$. We say $M \in \Delta_{2}$, if $M(2 u) \leq$ $K M(u)$ for all large $u$, where $K>2$ is a previously constant. $M(u) \in \nabla_{2}$ means that $N(v) \in \Delta_{2}$. Suppose that $(G, \Sigma, \mu)$ is a nonatomic finite measure space. For each $\Sigma$-measurable function $x(t)$ on $G$, we denote its mordular by

$$
\rho_{M}(x)=\int_{G} M(x(t)) d \mu .
$$

The set

$$
L_{M}=\left\{x(t): \rho_{M}(\lambda x)<\infty \text { for some } \lambda>0\right\}
$$

endowed with Luxemburg norm

$$
\|x\|=\inf \left\{\lambda>0, \rho_{M}(x / \lambda) \leq 1\right\}
$$

and Orlicz norm

$$
\|x\|^{\circ}=\inf \left\{k^{-1}\left[1+\rho_{M}(k x)\right]: k>0\right\}
$$

we denote by $L_{M}, L_{M}{ }^{\circ}$ respectively, and we call them Orlicz Spaces.

It is known that for all $x \neq 0$, there exists $k_{x}>0$ such that

$$
\|x\|^{\circ}=k_{x}^{-1}\left(1+\rho_{M}\left(k_{x} x\right)\right) .
$$

First we give some auxiliary Lemmas.

Lemma 1. Any $f \in L_{M}$ has the unique decomposition

$$
f=v+\phi \quad\left(v \in L_{N}{ }^{o}, \phi \text { is a singular functional }\right), \text { i.e. }
$$




$$
f(x)=\int_{G} x(t) v(t) d t+\phi(x) \quad\left(x \in L_{M}\right) .
$$

Proof. See [4].

Lemma 2. $f=v+\phi$ is a support functional at $x \in L_{M} \backslash\{0\}$ iff

(1) $\rho_{M}\left(\frac{x}{\|x\|}\right)=1$

(2) $\|\phi\|=\phi\left(\frac{x}{\|x\|}\right)$,

(3) $x(t) v(t) \geq 0$ and for any (equivalently for some) $k \in K(v)$,

$$
p_{-}\left(\frac{|x(t)|}{\|x\|}\right) \leq k|v(t)| \leq p\left(\frac{|x(t)|}{\|x\|}\right)
$$

Proof. See [4].

Lemma 3. If $M \in \nabla_{2},[a, b]$ is an affine interval of $M(u)$, and $M(u)$ is neither linear on $[a-\varepsilon, a]$, nor on $[b, b+\varepsilon]$ for any $\varepsilon>0$, then for any $\varepsilon>0$, there exists $\delta>0$ such that for any $v \in[a, b]$,

$$
\frac{M(u)+M(v)}{2}-M\left(\frac{u+v}{2}\right)<\delta \text { implies } u \in[a-\varepsilon, b+\varepsilon]
$$

Proof. See [1].

Lemma 4. Assume $x \in S\left(L_{M}\right)$. If $\theta(x)=\inf \left\{c>0: \rho_{M}(x / c)<\right.$ $\infty\}<1$, then all support functionals of $x$ are in $L_{N^{\circ}}$.

Proof. See Lemma 2 in [5].

Theorem. $x \in S(X)$ is $W M$ point if and only if

(1) there exists $\tau>0$ such that $\rho_{M}((1+\tau) x)<\infty$.

(2) $\mu\{t \in G:|x(t)| \in(a, b]\}=0$ or $M \in \nabla_{2}$, where $[a, b]$ is an arbitrary structural affine interval of $M(u)$. 
(3) For any common end-point of two neighbour affine intervals $c, \mu\{t \in G:|x(t)|=c\}=0$.

Proof. Necessity. Without loss of generality, we assume $x(t) \geq 0$. If (1) is not true, then for any $\varepsilon>0, \rho_{M}((1+\varepsilon) x)=\infty$. Take $0<c<d$ such that $\mu E=\mu\{t: c \leq x(t) \leq d\}>0$. Put

$$
y=-\left.x\right|_{E}+\left.x\right|_{G \backslash E}
$$

Obviously $\|y\|=\|x\|=1$. For any $\varepsilon>0$, since $\rho_{M}\left(\frac{x+y}{2}\right)=\rho_{M}\left(\left.x\right|_{G \backslash E}\right)$ $<1$ and $\rho_{M}\left((1+\varepsilon) \frac{x+y}{2}\right)=\rho_{M}\left(\left.(1+\varepsilon) x\right|_{G \backslash E}\right)=\infty$, we deduce $\left\|\frac{x+y}{2}\right\|=$ 1 i.e. $\|x+y\|=2$.

Denote $G_{n}=\{t \in G:|x(t)| \leq n\}, x_{n}(t)=\left.x(t)\right|_{G_{n}}$. Clearly, $\left\|x_{n}\right\| \leq 1,\left\|x_{n}+x\right\| \rightarrow\|2 x\|=2$. We consider the sequence

$$
x_{1}, y, x_{2}, y, x_{3}, y, \cdots
$$

For any support functional $f=v+\phi$ at $x$, if $\phi(x) \neq 0$, then

$$
f\left(x_{n}\right)=\int_{G} x_{n}(t) v(t) d t \rightarrow \int_{G} x(t) v(t) d t=f(x)-\phi(x) \neq f(x)=1
$$

and if $\phi(x)=0$, then by Lemma 2 ,

$$
f(x-y)=\int_{E} 2 x(t) v(t) d t=\frac{1}{k_{v}} \int_{E} 2 x(t) k_{v} v(t) d t \geq \frac{1}{k_{v}} 2 c p_{-}(c) \mu E>0
$$

so $f(y) \neq f(x)=1$ and denoting the sequence by $\left(Z_{n}\right)$, we have $Z_{n} \in$ $B\left(L_{M}\right),\left\|x+Z_{n}\right\| \rightarrow 2$ and $f\left(Z_{n}\right) \neq 1$ for any support functional at $x$, i.e. $x$ is not WM point.

If (2) does not hold, then there exists an affine interval $[a, b]$ of $M(u)$, such that $\mu\{t \in G: x(t) \in(a, b]\}>0$ and there is a sequence 
$\left(u_{n}\right)$ such that $u_{n} \uparrow \infty$, and

$$
M\left(\frac{u_{n}}{2}\right)>\left(1-\frac{1}{n}\right) \frac{M\left(u_{n}\right)}{2} .
$$

Take $\varepsilon>0$ such that $\mu E=\mu\{t \in G: x(t) \in[a+\varepsilon, b]\}>0$ and measurable sets $E_{n} \subset E$ satisfying

$$
\rho_{M}\left(\left.x\right|_{G \backslash E}\right)+\int_{E} M(x(t)-\varepsilon) d t+M\left(u_{n}\right) \mu\left(E \backslash E_{n}\right)=1
$$

Then $\mu\left(E \backslash E_{n}\right) \rightarrow 0$ and $\mu E_{n} \rightarrow \mu E$. Let

$$
y_{n}=\left.x\right|_{G \backslash E}+\left.(x(t)-\varepsilon)\right|_{E_{n}}+\left.u_{n}\right|_{E \backslash E_{n}} .
$$

Then $\left\|y_{n}\right\|=1$ and

$$
\begin{aligned}
\rho_{M}\left(\frac{x+y_{n}}{2}\right) & =\rho_{M}\left(\left.x\right|_{G \backslash E}\right)+\rho_{M}\left(\left.\frac{x+x-\varepsilon}{2}\right|_{E_{n}}\right) \\
& +M\left(\frac{u_{n}}{2}\right) \mu\left(E \backslash E_{n}\right) \\
& >\rho_{M}\left(\left.x\right|_{G \backslash E}\right)+\frac{1}{2} \rho_{M}\left(\left.x\right|_{E_{n}}\right)+\frac{1}{2} \rho_{M}\left(\left.(x-\varepsilon)\right|_{E_{n}}\right) \\
& +\left(1-\frac{1}{n}\right) \frac{M\left(u_{n}\right)}{2} \mu\left(E \backslash E_{n}\right) \\
& >\frac{1}{2}\left(1-\frac{1}{n}\right)\left(\rho_{M}\left(y_{n}\right)+\rho_{M}\left(\left.x\right|_{G \backslash\left(E \backslash E_{n}\right)}\right) \rightarrow 1\right.
\end{aligned}
$$

whence $\left\|x+y_{n}\right\| \rightarrow 2$. 
Take any support functional $f=v+\phi$ at $x$. Since $\rho_{M}((1+\tau) x)<\infty$ for some $\tau>0$, we have $\theta(x) \leq \frac{1}{1+\tau}<1$. By Lemma 4, we get that all support functionals at $x$ are in $L_{N}{ }^{\circ}$. Therefore

$$
\begin{aligned}
f\left(x-y_{n}\right) & =<x-y_{n}, v>=\int_{E_{n}} \varepsilon v(t) d t-\int_{E \backslash E_{n}} u_{n} v(t) d t \\
& +\int_{E \backslash E_{n}} x(t) v(t) d t \\
& \geq \int_{E_{n}} \varepsilon v(t) d t-\int_{E \backslash E_{n}} u_{n} v(t) d t \\
& \geq \frac{1}{k_{v}}\left(\varepsilon p(a) \mu E_{n}-\left\|y_{n}\right\| p(b)\left\|\chi_{E \backslash E_{n}}\right\|_{N}{ }^{\circ}\right) \\
& \rightarrow \frac{1}{k_{v}} \varepsilon p(a) \mu E>0 .
\end{aligned}
$$

This implies $f\left(y_{n}\right) \neq f(x)=1$ which gives that $x$ is not a WM point. This is a contradiction.

The proof of (3) is trivial, it is omited.

Sufficiency. Let $x_{n} \in B(X)$ and $\left\|x_{n}+x\right\| \rightarrow 2$.

First we will prove that

$$
\rho_{M}\left(x_{n}\right) \rightarrow 1, \rho_{M}\left(\frac{x_{n}+x}{2}\right) \rightarrow 1
$$

For any $\varepsilon>0$ and $n$ large enough we have $\left\|(1+\varepsilon) \frac{x_{n}+x}{2}\right\|>1$. Then 


$$
\begin{aligned}
& 1<\rho_{M}\left((1+\varepsilon) \frac{x_{n}+x}{2}\right)=\rho_{M}\left(\frac{1+\varepsilon}{2} x_{n}+\frac{1-\varepsilon}{2} \frac{1+\varepsilon}{1-\varepsilon} x\right) \\
& \leq \frac{1+\varepsilon}{2} \rho_{M}\left(x_{n}\right)+\frac{1-\varepsilon}{2} \rho_{M}\left(\frac{1+\varepsilon}{1-\varepsilon} x\right)
\end{aligned}
$$

Take $\varepsilon$ small enough that $\frac{1+\varepsilon}{1-\varepsilon}<1+\tau$. By (1), we have

$$
1 \leq \frac{1+\varepsilon}{2} \rho_{M}\left(x_{n}\right)+\frac{1-\varepsilon}{2}\left(\rho_{M}(x)+o(\varepsilon)\right)
$$

Since $\varepsilon$ is arbitrary, we get $\rho_{M}\left(x_{n}\right) \rightarrow 1$ immediately.

Similarly, by $\left\|\frac{x_{n}+x}{2}+x\right\| \rightarrow 2$, we can deduce that $\rho_{M}\left(\frac{x_{n}+x}{2}\right) \rightarrow 1$.

We will discuss two cases.

I. For any affine interval $(a, b]$ of $M(u), \mu\{t: x(t) \in(a, b]\}=0$.

First we will prove that $x_{n}-x \stackrel{\mu}{\rightarrow} 0$.

Denote by $\left\{a_{i}\right\}$ the left endpoints of all structural affine intervals.

$G_{a}=\left\{t \in G: x(t) \in\left\{a_{i}\right\}_{i=1}^{\infty}\right\}$. If $x_{n}-x \stackrel{\mu}{\rightarrow} 0$ on $G \backslash G_{a}$ is not true, then there exists $\varepsilon, \sigma>0$ such that

$$
\mu\left\{t \in G \backslash G_{a}:\left|x_{n}(t)-x(t)\right| \geq \varepsilon\right\} \geq \sigma .
$$

By $1 \leftarrow \rho_{M}\left(x_{n}\right) \geq M(D) \mu\left\{t:\left|x_{n}(t)\right|>D\right\}$, we can take $D$ large enough such that

$$
\mu\left\{t:\left|x_{n}(t)\right|>D\right\}<\frac{\sigma}{4}, \mu\{t:|x(t)|>D\}<\frac{\sigma}{4} .
$$

Hence

$$
\mu\left\{t \in G \backslash G_{a}:\left|x_{n}(t)-x(t)\right| \geq \varepsilon,|x(t)|,\left|x_{n}(t)\right| \leq D\right\} \geq \frac{\sigma}{2} .
$$


Let $r_{1}, r_{2}, \ldots, r_{i}, \ldots$, be endpoints of all structural affine intervals of $M(u)$, then $\mu\left\{t \in G \backslash G_{n}: x(t)=r_{i}\right\}=0$. So there exists a neighbourhood $V_{i}$ of $r_{i}$ small enough such that $\mu\left\{t \in G \backslash G_{a}: x(t) \in\right.$ $\left.V_{i}\right\}<\frac{\sigma}{4 \cdot 2^{i}}$. Therefore

$$
\mu\left\{t: x(t) \in \bigcup_{i=1}^{\infty} V_{i}\right\}<\frac{\sigma}{4}
$$

Thus $\mu G_{n}>\frac{\sigma}{4}$ where

$$
\begin{array}{r}
G_{n}=\left\{t \in G \backslash G_{a}:\left|x_{n}(t)-x(t)\right| \geq \varepsilon,|x(t)|,\left|x_{n}(t)\right| \leq D,\right. \\
\left.x(t) \in S_{M} \backslash \bigcup_{i=1}^{\infty} V_{i}\right\} .
\end{array}
$$

It is easy to know that there exists $\delta, 0<\delta<1$, such that for any $t \in G_{n}$

$$
M\left(\frac{x_{n}(t)+x(t)}{2}\right) \leq(1-\delta)\left(\frac{M\left(x_{n}(t)\right)+M(x(t))}{2}\right)
$$

We have a contradiction

$$
\begin{aligned}
0 & \leftarrow\left(\frac{\rho_{M}\left(x_{n}\right)+\rho_{M}(x)}{2}-\rho_{M}\left(\frac{x_{n}(t)+x(t)}{2}\right)\right. \\
& =\int_{G}\left(\frac{M\left(x_{n}(t)\right)+M(x(t))}{2}-M\left(\frac{x_{n}(t)+x(t)}{2}\right)\right) d t \\
& \geq \int_{G_{n}} \frac{\left(\frac{M\left(x_{n}(t)\right)+M(x(t))}{2}-M\left(\frac{x_{n}(t)+x(t)}{2}\right)\right) d t}{2} \\
& \geq \delta \int_{G_{n}} \frac{\frac{M\left(x_{n}(t)\right)+M(x(t))}{2} d t}{2}
\end{aligned}
$$




$$
\geq \frac{\delta}{2} M\left(\frac{\varepsilon}{2}\right) \frac{\sigma}{4} .
$$

Using the same method as above, we get for any $\varepsilon>0$,

$$
\mu\left\{t \in G_{a}: x_{n}(t) \leq x(t)-\varepsilon\right\} \rightarrow 0 .
$$

Combining this with $x_{n}-x \stackrel{\mu}{\rightarrow} 0$ on $G \backslash G_{a}$, we deduce that for any $E \subset G$.

$$
\lim _{n \rightarrow \infty} \inf \rho_{M}\left(\left.x_{n}\right|_{E}\right) \geq \rho_{M}\left(\left.x\right|_{E}\right)
$$

If $x_{n}-x \stackrel{\mu}{\rightarrow} 0$ on $G_{a}$ is not true, then there exists positive numbers $a, \varepsilon$ and $\sigma$ such that $\mu E_{a}=\mu\left\{t \in G_{a}: x_{n}(t)-x(t)=x_{n}(t)-a \geq \varepsilon\right\} \geq \sigma$. Thus we can deduce easily

$$
\lim _{n \rightarrow \infty} \inf \rho_{M}\left(x_{n} \mid E_{a}\right)>\rho_{M}\left(\left.x\right|_{E_{a}}\right)
$$

Combining this with (1), we get a contradiction

$$
1=\lim _{n \rightarrow \infty} \rho_{M}\left(x_{n}\right)>\rho_{M}(x)=1
$$

This finishes the proof that $x_{n}-x \stackrel{\leftrightarrow}{\rightarrow} 0$.

Take any support functional $f$ at $x$. By the assumption (1), we have $f=v \in S\left(L_{N}{ }^{\circ}\right)$. Now we will prove $\left\langle x_{n}, v\right\rangle \rightarrow 0$ or $\left\langle x_{n}-x, v\right\rangle \rightarrow 0$. For any $\varepsilon>0$, there exists $\delta>0$ such that for any $e \subset G$, $\mu e<\delta$ implies $\rho_{N}\left(\left.v\right|_{e}\right)<\varepsilon$ and $\rho_{M}\left(\left.x\right|_{e}\right)<\varepsilon$. Since $x_{n}-x \stackrel{\mu}{\rightarrow} 0$, there exists $F \subset G$ with $\mu(G \backslash F)<\delta$ such that $x_{n}-x \rightarrow 0$ uniformly on $F$.

So there exists $n_{0},\left|x_{n}(t)-x(t)\right|<\varepsilon(t \in F)$, and

$$
\left|\int_{F}\left(M\left(x_{n}(t)\right)-M(x(t))\right) d t\right|<\varepsilon \quad \text { as } n \geq n_{0} .
$$


Noticing $\rho_{M}\left(x_{n}\right) \rightarrow 1=\rho_{M}(x)$, we have $\mid \int_{G \backslash F}\left(M\left(x_{n}(t)-M(x(t)) d t \mid<\right.\right.$ $\varepsilon$ for $n$ large enough. So

$$
\int_{G \backslash F} M\left(x_{n}(t)\right) d t<\int_{G \backslash F} M(x(t)) d t+\varepsilon<2 \varepsilon \text { for } n \text { large enough. }
$$

Thus

$$
\begin{aligned}
<x_{n}-x, v> & =\int_{F}\left(x_{n}(t)-x(t)\right) v(t) d t+\int_{G \backslash F}\left(x_{n}(t)-x(t)\right) v(t) d t \\
& \leq \varepsilon\|1\|+\rho_{M}\left(\left.x_{n}\right|_{G \backslash F}\right)+\rho_{M}\left(\left.x\right|_{G \backslash F}\right)+2 \rho_{N}\left(\left.v\right|_{G \backslash F}\right) \\
& =0(\varepsilon) .
\end{aligned}
$$

By the arbitrariness of $\varepsilon$ we get $\left\langle x_{n}-x, v>\rightarrow 0\right.$

II. $M \in \nabla_{2}$, First we will prove that

$$
\lim _{\mu e \rightarrow 0} \sup _{n} \rho_{M}\left(\left.x_{n}\right|_{e}\right)=0
$$

If it is not true, then there exists $\varepsilon>0$ and $e_{n}, \mu e_{n} \downarrow 0$ with $\rho_{M}\left(x_{n} \mid e_{n}\right) \geq$ $\varepsilon$.

Without loss of generality, we may assume $\left|x_{n}(t)\right| \geq u_{0}>0\left(t \in e_{n}\right)$.

Let $1+\dot{\tau}=\frac{1}{1-\tau^{\prime}}\left(\tau^{\prime}>0\right)$. Since $M \in \nabla_{2}$, there exists $\delta>0$ such that $M\left(\frac{u}{1+\tau^{\prime}}\right) \leq(1-\delta) \frac{M(u)}{1+\tau^{\prime}}\left(u \geq u_{0}\right)$.

By $\rho_{M}\left(\frac{x}{1-\tau^{\prime}}\right)<\infty$, we have $\rho_{M}\left(\frac{x}{1-\tau^{\prime}},\left.\right|_{e n}\right)<\frac{\delta \varepsilon}{2}$ as $n$ is large enough. Hence 


$$
\begin{aligned}
\int_{e_{n}} M & \left(\frac{x_{n}(t)+x(t)}{2}\right) d t=\int_{e_{n}} M\left(\frac{1+\tau^{\prime}}{2} \frac{x_{n}(t)}{1+\tau^{\prime}}+\frac{1-\tau^{\prime}}{2} \frac{x(t)}{1-\tau^{\prime}}\right) d t \\
& \leq \frac{1+\tau^{\prime}}{2} \int_{e_{n}} M\left(\frac{x_{n}(t)}{1+\tau^{\prime}}\right) d t+\frac{1-\tau^{\prime}}{2} \int_{e_{n}} M\left(\frac{x(t)}{1-\tau^{\prime}}\right) d t \\
& \leq \frac{1+\tau^{\prime}}{2}(1-\delta) \frac{1}{1+\tau^{\prime}} \int_{e_{n}} M\left(x_{n}(t)\right) d t+\frac{\delta \varepsilon}{4} \\
& \leq \frac{1}{2} \int_{e_{n}} M\left(x_{n}(t) d t-\frac{\delta \varepsilon}{2}+\frac{\delta \varepsilon}{4}\right. \\
& =\frac{1}{2} \int_{e_{n}} M\left(\bar{x}_{n}(t)\right) d t-\frac{\delta \varepsilon}{4} .
\end{aligned}
$$

Therefore, we get a contradiction

$$
\begin{aligned}
1 & \left.\leftarrow \frac{x_{n}+x}{2}\right)=\rho_{M}\left(\left.\frac{x_{n}+x}{2}\right|_{G \backslash e_{n}}\right)+\rho_{M}\left(\frac{x_{n}+x}{2}-e_{n}\right) \\
& \leq \frac{\rho_{M}\left(\left.x_{n}\right|_{G \backslash e_{n}}\right)+\rho_{M}\left(\left.x\right|_{G \backslash e_{n}}\right)}{2}+\frac{1}{2} \rho_{M}\left(\left.x_{n}\right|_{e_{n}}\right)-\frac{\delta \varepsilon}{4} \\
& \rightarrow 1-\frac{\delta \varepsilon}{4} .
\end{aligned}
$$

Denote by $\left\{b_{i}\right\}$ the right endpoints of the structural affine intervals.

Put $v(t)=\frac{1}{k} u(t)$, where

$$
u(t)=\left\{\begin{array}{ll}
p_{-}(x(t)) & x(t) \in\left\{b_{i}\right\}_{i=1}^{\infty} \\
p(x(t)) & \text { otherwise }
\end{array} \text { and } k=\|u\|_{N}^{o}\right.
$$


It is easy to prove $k_{v}=k$. By Lemma 2, we know $v \in S\left(L_{N}{ }^{\circ}\right)$ is a support functional of $x$.

Now we prove that $\left\langle x_{n}, v\right\rangle \rightarrow 1$.

Let $\left\{\left[a_{i}, b_{i}\right]\right\}_{i}$ be all structural affine intervals of $M(u)$, and $E_{i}=$ $\left\{t \in G: x(t) \in\left[a_{i}, b_{i}\right]\right\}$.

By assumption (3) $E_{i} \cap E_{j}=\phi(i \neq j)$.

For any $\varepsilon>0$, there exists $d$, such that $e \subset G, \mu e<d$ imply

$$
\rho_{M}\left(\left.x\right|_{e}\right)<\varepsilon, \rho_{N}\left(\left.k v\right|_{e}\right)<\varepsilon \text { and } \rho_{M}\left(\left.x_{n}\right|_{e}\right)<\varepsilon
$$

Recall that the last inequality holds uniformly with respect to $n$ by $M \in$ $\nabla_{2}$. Since $\sum_{i=1}^{\infty} \mu E_{i} \leq \mu G<\infty$, there exists $m$ such that $\mu\left(\bigcup_{i>m} E_{i}\right)<d$. For each $i \leq m$ and all $u \in\left[a_{i}, b_{i}\right]$, we have

$$
u p\left(a_{i}\right)=M(u)+N\left(p\left(a_{i}\right)\right) .
$$

Therefore, we can find $\beta>0$ such that if $u \in\left[a_{i}-\beta, b_{i}+\beta\right]$, then

$$
u p\left(a_{i}\right)>M(u)+N\left(p\left(a_{i}\right)\right)-\varepsilon \quad(i=1,2, \ldots, m)
$$

By Lemma 3, there exists $\delta>0$ such that

$$
\frac{M(u)+M(w)}{2}-\frac{M(u+w)}{2}<\delta \quad \text { and } w \in\left[a_{i}, b_{i}\right]
$$

imply

$$
u \in\left[a_{i}-\beta, b_{i}+\beta\right] \quad(i=1,2, \ldots, m)
$$

Since $\int_{G}\left(\frac{M\left(x_{n}(t)\right)+M(x(t))}{2} \_M\left(\frac{x_{n}(t)+x(t)}{2}\right)\right) d t \rightarrow 0$, we get

$$
\frac{M\left(x_{n}(t)\right)+M(x(t))}{2}-M\left(\frac{x_{n}(t)+x(t)}{2}\right) \stackrel{\mu}{\rightarrow} 0
$$


Denote $F_{n}=\left\{t \in \bigcup_{i=1}^{m} E_{i}: \frac{M\left(x_{n}(t)\right)+M(x(t))}{2}-M\left(\frac{x_{n}(t)+x(t)}{2}\right) \geq \delta\right\}$, by (5), we have $\mu F_{n}<d$ for $n$ large enough. For $t \in \bigcup_{i=1}^{m} E_{i} \backslash F_{n}$, we have $x(t) \in \bigcup_{i=1}^{m}\left[a_{i}, b_{i}\right]$ and

$$
\frac{M\left(x_{n}(t)\right)+M(x(t))}{2}-M\left(\frac{x_{n}(t)+x(t)}{2}\right)<\delta .
$$

By (4) $x_{n}(t) \in\left[a_{i}-\beta, b_{i}+\beta\right]$, and by (3) we get

$$
x_{n}(t) p\left(a_{i}\right)>M\left(x_{n}(t)\right)+N\left(p\left(a_{i}\right)\right)-\varepsilon .
$$

Notice that $k v(t)=u(t)=p\left(a_{i}\right)$, so

$$
x_{n}(t) k v(t)>M\left(x_{n}(t)\right)+N(k v(t))-\varepsilon \quad\left(t \in \bigcup_{i=1}^{m} E_{i} \backslash F_{n}\right)
$$

Denote $E_{0}=G \backslash \bigcup_{i=1}^{\infty} E_{i}$. Using the same method as in the case I, we get $x_{n}-x \stackrel{\mu}{\rightarrow} 0$ on $E_{0}$. Thus, there exists $F_{0} \subset E_{0}, \mu F_{0}<d$ such that

$$
\left|x_{n}(t)-x(t)\right|<\varepsilon,\left|M\left(x_{n}(t)\right)-M(x(t))\right|<\varepsilon \text { uniformly on } E_{0} \backslash F_{0}
$$

for $n$ large enough. Hence

$$
\begin{aligned}
x_{n}(t) k v(t) & >(x(t)-\varepsilon) k v(t)=M(x(t))+N(k v(t))-\varepsilon k v(t) \\
& >M\left(x_{n}(t)\right)+N(k v(t))-\varepsilon-\varepsilon k v(t) \quad\left(t \in E_{0} \backslash F_{0}\right)
\end{aligned}
$$

It follows from (6) and (7) that 


$$
\begin{aligned}
& <x_{n}, k v>=\int_{\bigcup_{i=1}^{m} E_{i} \backslash F_{n}}+\int_{E_{0} \backslash F_{0}}+\int_{F_{n}}+\int_{\bigcup_{i>m} E_{i}}+\int_{F_{0}} x_{n}(t) k v(t) d t
\end{aligned}
$$

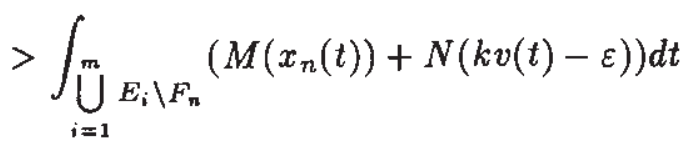

$$
\begin{aligned}
& +\int_{E_{0} \backslash F_{0}}\left(M\left(x_{n}(t)\right)+N(k v(t))-\varepsilon-\varepsilon k v(t)\right) d t \\
& -\int_{F_{n}}-\int_{\bigcup_{i>m} E_{i}}-\int_{F_{0}}\left(M\left(x_{n}(t)\right)+N(k v(t))\right) d t \\
& >\int_{\left(\bigcup_{i=1}^{m} E_{i} \backslash F_{n}\right) \cup E_{0} \backslash F_{0}}\left(M\left(x_{n}(t)\right)+N(k v(t))\right) d t \\
& -\varepsilon \mu G-\varepsilon k \||| 1 \mid-6 \varepsilon \\
& =\int_{G}\left(M\left(x_{n}(t)\right)+N(k v(t)) d t\right.
\end{aligned}
$$

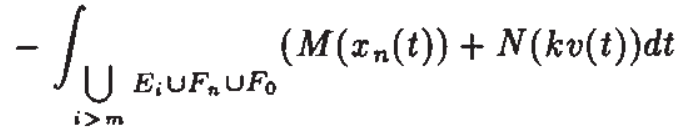

$$
\begin{aligned}
& -\varepsilon \mu G-\varepsilon k\|1\|-6 \varepsilon \\
& \rightarrow 1+\rho_{N}(k v)-o(\varepsilon)=k\|v\|_{N}{ }^{\circ}-o(\varepsilon)=k-o(\varepsilon) .
\end{aligned}
$$

This shows that $\left\langle x_{n}, v\right\rangle \rightarrow 1$. 
Corollary $L_{M}$ has WM property if and only if

(1) $M \in \Delta_{2}$

(2) $M \in S C$ or $M \in \nabla_{2}$

(3) There are not two neighbour affine intervals of $M(u)$.

Proof. Sufficiency. Take any $x \in S\left(L_{M}\right)$. Since $M \in \Delta_{2}, L_{M}=$ $E_{M}$. So for any $\tau>0, \rho_{M}((1+\tau) x)<\infty$.

$M \in S C$ implies $M(u)$ has no any affine interval i.e.

$\{t \in G: x(t) \in(a, b]\}=\Phi$, then $\mu\{t \in G: x(t) \in(a, b]\}=0$

By Theorem, we get $x$ is WM point.

If $M \in \nabla_{2}$ combining $\rho_{M}((1+\tau) x)<\infty$ and by Theorem, we get that $x$ is WM point. So $L_{M}$ has WM property.

Necessity. If $M \notin \Delta_{2}$, we may construct $x \in S\left(L_{M}\right)$ with $\rho_{M}((1+$ $\tau) x)=\infty$, for any $\tau>0$. By Theorem, $x$ is not WM point.

If $M \notin S C$, and $M \notin \nabla_{2}$. Then $M(u)$ has an affine interval $[a, b]$. Take $d$ large enough, and measurable $E, F \subset G, E \cap F=\Phi$ such that

$$
M(b) \mu E+M(d) \mu F=1
$$

Put $x=\left.b\right|_{E}+\left.d\right|_{F}$. Then $x \in S\left(L_{M}\right)$ but $\mu\{t \in G: x(t) \in(a, b]\} \geq$ $\mu E>0$.

By Theorem, $x$ is not WM point.

If $[a, c],[c, b]$ are the two neighbour structural affine intervals of $M(u)$, take $d$ large enough, $E, F \subset G, E \cap F=\Phi$ satisfying

$$
M(c) \mu E+M(d) \mu F=1
$$

Let $x=\left.c\right|_{E}+\left.d\right|_{F}$. Then $x$ is not WM point.

\section{References}

[1] Chen Shutao and Duan Yanzheng, WM property of Orlicz spaces, Northeastern Math. J. 8(4), (1992), 498-502. 
[2] Chen Shutao, Lu Yanming and Wang Baoxiang, WM property and LKR of Orlicz sequence spaces, Fasciculi, Math. 4(1991), 19-25.

[3] Duan Yanzheng, Several Structural Problems of Orlicz spaces, Dissertation of Ph.D, HIT. 1990.

[4] Wu C. and Wang T., Orlicz spaces and its applications. Heilongjiang Sci \& Tech. press, 1983, Harbin.

[5] Grzaslewicz, R. and Hudzik, H., Smooth points of Orlicz spaces equipped with Luxemburg norm. Math. Nachr. 155(1992), 31-45.

[6] Shutao Chen, Huizik, H. and Anna Kaminska, Support functionals and smooth points in Orlicz function spaces equipped with the Orlicz norm, Math. Japonica. 39(2)(1994), 271-279.

Wang Tingfu, Hao Cuixia, Li Minli Harbin. Univ. Sci. Tech. Box 610.

Harbin 150080, P.R. China.
Recibido: 11 de Mayo de 1994

Revisado:9 de Enero de 1995 